TITLE:

\title{
Immediate impact of the COVID-19 pandemic on the socio-emotional and digital skills of Japanese children
}

\section{$\operatorname{AUTHOR}(\mathrm{S}):$}

Moriguchi, Yusuke; Sakata, Chifumi; Meng, Xianwei; Todo, Naoya

\section{CITATION:}

Moriguchi, Yusuke ...[et al]. Immediate impact of the COVID-19 pandemic on the socio-emotional and digital skills of Japanese children. PsyArXiv 2020

ISSUE DATE:

2020-05-28

URL:

http://hdl.handle.net/2433/263908

\section{RIGHT:}

This article is a preprint and has not been certified by peer review.; The copyright holder for this preprint is the author/funder, who has granted [PsyArXiv] a license to display the preprint in perpetuity. It is made available under a CC-BY 4.0 International license.; This is not the published version. Please cite only the published version. この論文は出 版社版でありません。引用の際には出版社版をご確認ご利用ください。 


\section{Did the COVID-19 Pandemic Have Immediate Impacts on the Socio- Emotional Behaviors of Japanese Children}

1 Yusuke Moriguchi ${ }^{1 *}$, Chifumi Sakata ${ }^{1}$, Xianwei Meng ${ }^{2}$, Naoya Todo ${ }^{3}$

$2 \quad{ }^{1}$ Graduate School of Letters, Kyoto University, Yoshidahoncho, Kyoto, Japan

$3 \quad{ }^{2}$ Center for Baby Science, Doshisha University, Kizugawadai, Kizugawa, Kyoto, Japan

$4 \quad{ }^{3}$ Faculty of Human Sciences, University of Tsukuba, Tsukuba, Japan

5 * Correspondence:

6 Yusuke Moriguchi

7 moriguchi.yusuke.8s@kyoto-u.ac.jp

8 Keywords: COVID-19, pandemic 2 , child development 3 , socio-emotional skill 4 prosocial

9 behavior5

\section{Abstract}

12 A novel coronavirus, SARS-CoV-2, has spread widely throughout the world. To reduce the spread of 13 infection, children are prevented from going to school and have fewer opportunities for in-person 14 communication. Although the pandemic has impacted the everyday lives of children, its impact on their development is unknown. This cross-sectional study compared Japanese children's socioemotional behaviors before and during the pandemic. Parents completed a web-based questionnaire before and during the pandemic for children ages 4-9. Children's socio-emotional development in an everyday context was assessed using the Strengths and Difficulties Questionnaire (SDQ). The results indicated that during the pandemic, children were more prosocial and experienced more problems in their peer relationships, but no differences were found in emotional symptoms, conduct problems, hyperactivity between before and during the pandemic. Overall, our results suggest the pandemic may have immediate impact on children's socio-emotional behaviors. We discussed the results in terms of behavioral immune system.

Trial registration: We pre-registered our hypotheses, method, primary analyses, and sample size (https://osf.io/c7p6b)

\section{Introduction}

A novel coronavirus, SARS-CoV-2, has now spread widely throughout the world. Due to the virus' high transmission rate, relatively long incubation period, and increased mortality rate in people with certain conditions (e.g., older people), the World Health Organization (WHO) has provided guidelines to help prevent the public from becoming infected with the virus (World Health Organization, 2020). Common strategies include asking or ordering people to stay at home, avoid crowds or large gatherings, and practice social distancing. Consequently, people in several countries have been prevented from going to work or school including kindergarten and have fewer opportunities for in-person communication with others. The clinical course of the coronavirus 
disease, COVID-19, appears to be relatively mild in children compared to other populations (Pathak et al., 2020; Wu \& McGoogan, 2020), although infants were found to be at high risk of becoming severely or critically ill (Dong et al., 2020). Nevertheless, the effects of the societal changes implemented to decrease the likelihood of SARS-CoV-2 infection on children's cognitive, social, and emotional development are unknown. According to Bronfenbrenner (Bronfenbrenner, 1979), child development is a function of the interaction between several systems and includes culture, parental occupations, schooling, peer relationships, and parenting. Thus, changes in one system can directly or indirectly affect children's development. In the case of COVID-19, the pandemic can affect parents' work, and children's schooling by both children and adults, which may in turn have significant effects on children's cognitive, social, and emotional development.

Although several psychological studies have examined the effect of the COVID-19 pandemic on children's mental health (Golberstein et al., 2020; Orgilés et al., 2020; Pisano et al., 2020; Xie et al., 2020), it remains unclear whether the pandemic affects children's socio-emotional behaviors. One study in Italy and Spain reported that children experienced emotional and behavioral problems (e.g., difficulty concentrating) after the pandemic outbreak (Orgilés et al., 2020), but this study did not assess the population before the pandemic and thus the change in emotional problems could not be compared. Therefore, the present study examined differences in children's socio-emotional behaviors before and during the pandemic.

We assessed whether children's socio-emotional behaviors (emotional symptoms, conduct problems, hyperactivity, peer problems, and prosocial behavior) differed by their experience of the pandemic by comparing their socio-emotional behaviors before and during the pandemic. In terms of prosocial behavior, a previous study reported that experiencing a natural disaster (an 8.0 magnitude earthquake in China) affected children's prosocial behaviors. The researchers compared the prosocial behaviors of two groups: a group 6- and 9-year-old Chinese children who lived near the epicenter of the earthquake before the disaster and a second group of Chinese children matched by age and school of attendance after the earthquake. The results suggested that 6-year-old children became more selfish whereas 9-year-old children became more prosocial immediately after the disaster. Although the COVID-19 pandemic may be different from the earthquake in several ways, e.g., children may feel more anxiety about being infected by the virus, the results from the previous study suggest that experiencing an adversity can have differential effects by age on children's prosocial behaviors. Thus, we hypothesized that children would experience differences in their social relationships during the pandemic that would show the differences in their socio-emotional behaviors. Moreover, based on the previous study, we expected children's age to moderate the effect of the pandemic on prosocial behaviors. To assess the differences in social relationships, we assessed the durations for children's schooling, outside play, and lessons (e.g., music, dance).

\section{$2 \quad$ Materials and Methods}

\subsection{Participants}

In Japan, the first infected person infected with SARS-CoV-2 was identified in January 2020, and the number of infected people has since increased although the growth rate was lower than in many other countries (Worldometer, 2020). On 27th February 2020, the government asked all schools across the country to close until March 2020, and the vast majority of schools complied (but nursery schools did not). School started to re-open at the beginning of April 2020, but the government declared a state of emergency covering seven prefectures including Tokyo and Osaka on 7th April 2020. Thus, most of the schools in seven prefectures closed whereas about $80 \%$ of the kindergartens and half of

This is a provisional file, not the final typeset article 
elementary schools in the other prefectures started to open on 10th April 2020 (Ministry of Education, Culture, Sports, Science and Technology, 2020). Subsequently, the declaration to close schools was extended to all regions on 16th April 2020, and most of the schools in all prefectures closed until 6th May 2020. Thus, data for the During-pandemic sample was collected when most of the schools were closed and children had less time for schooling and meeting with friends. For this study, we preregistered our hypotheses, method, primary analyses, and sample size (https://osf.io/c7p6b).

We conducted two cross-sectional studies in which we administered an internet-based survey to parents at two time periods, before and during the pandemic. Our Before-pandemic sample comprised primary caregivers of children ages 0-9 who were randomly selected from the population of a database (Cross Marketing Inc. Tokyo, Japan). The Before-pandemic sample completed the survey 26 - 30 September 2019. A total of 1215 participants completed the questionnaire, but 293 participants were excluded, of which 255 participants incorrectly answered trap questions and 38 participants inappropriately answered questions (e.g., participants who chose " 1 " in a series of questions). Out of 922 participants (who included parents of children ages 0-9), we assigned the first 70 participants in each age group (4 to 9 years of age) to this study (for a total 420 participants). Sample characteristics are presented in Table 1 and S1.

Our During-pandemic sample was selected in the same way as our Before-Pandemic sample. No participants were the same between the Before- and During- pandemic phases. The During-pandemic sample completed the survey 28 - 30 April 2020. During recruitment, 1045 participants completed the questionnaire, but 152 participants were excluded, of which 81 participants incorrectly answered trap questions and 71 participants answered inappropriately. After assigning parents to the studies, we had a total of 420 parents.

\subsection{Stimuli and Procedure}

The online questionnaire consisted of two parts. In the first part, parents were asked to complete background information about themselves and their children. In the second part, parents were given a questionnaire about their children's socio-emotional development and their social life.

\subsubsection{Background information}

In the first part, parents answered questions about their background. Background information included parental age, parental education, family size, children's age, children's sex, and children's sleep hours (when children get up and go to sleep). Parental education level was assigned a value from 1 to 5 ( $1=$ less than high school, $2=$ high school, $3=$ =some college, $4=$ =undergraduate degree, $5=$ graduate level).

\subsubsection{Socio-emotional behaviors}

In the second part, parents answered questions about their children's socio-emotional behaviors. Children's socio-emotional behaviors in an everyday context was assessed using the SDQ (Strengths and Difficulties Questionnaire) (Goodman, 1997; Matsuishi et al., 2008; Moriguchi et al., 2020). The SDQ is a screening measure of social, emotional, and behavioral functioning. The 25 -item SDQ is divided into five subscales, namely, emotional symptoms, conduct problems, hyperactivity, peer problems, and prosocial behavior. Emotion symptoms include five items, such as "Often complains of headaches, stomach-aches or sickness." Conduct problems include five items such as "Often fights with other children or bullies them." Hyperactivity includes five items such as "Restless, overactive, 
cannot stay still for long." Peer problems include five items, such as "Has at least one good friend." Prosocial behavior includes five items such as "Shares readily with other children, for example, toys, treats, pencils." The parents answered whether each item applied to a child on a three-point scale from 0 "not true" to 2 "certainly true."

\subsubsection{Social life}

126 To assess the differences in children's social lives, questions were asked regarding the duration of time that, children's schooling, children's outside play, and children's lessons (e.g., music, dance). We asked the number of days of children's schooling per week, and the average hours of outside play and lessons per day.

\section{$130 \quad 2.3 \quad$ Analytic plan}

Analyses were conducted in R (version 3.6,1). We conducted two analyses. First, we examined dependent variables that may be different before and during the pandemic. In our preregistration for the study, we planned to assess whether period and children's age affected their social life and socioemotional behaviors using a MANOVA. The analysis included period (Before-pandemic vs. Duringpandemic) and age (4 to 9) as independent variables and durations of, children's schooling, children's outside play and lessons, along with sub-scale scores for the SDQ as dependent variables. However, not all dependent variables were normally distributed, and we could not conduct the planned MANOVA. Instead, we conducted MANOVA within the framework of structural equation modelling (SEM). That is, we applied the MANOVA model to the data and estimated the parameters corresponding to the main effects using maximum likelihood estimation with robust (Huber-White) standard errors and a scaled test statistic that is (asymptotically) equal to the Yuan-Bentler test statistic using the "lavaan" package (Rosseel, 2012).

Second, we conducted a planned SEM analysis to assess the relationships between period and SDQ sub-scale scores, which were mediated by parents' and children's social lives. Specifically, we used variables indicative of children's and parent's social lives if we found significant main effects of period in the preceding analyses. We used background information as control variables for the analyses if we found significant differences in the Before-pandemic and During-pandemic samples.

\section{$148 \quad 3 \quad$ Results}

The descriptive data are reported in Table 1. Children's age in months, parental age, sex ratio (ratio of boys to girls), the number of family members, and parental education did not differ by period, Before-pandemic vs. During-pandemic. Children's sleeping time was higher During-pandemic than Before-pandemic $(\mathrm{t}(838)=-3.453, \mathrm{p}=.001, \mathrm{~d}=.24)$. Thus, Before-pandemic and During-pandemic samples were generally matched. We included demographic variables as control variables in our subsequent analyses.

First, we assessed whether period and children's age impacted children's socio-emotional behaviors and social lives. Period was significantly associated with children's peer problems $(\beta=0.264, p$ $=.033)$ as well as durations of, children's schooling $(\beta=-4.233, \mathrm{p}<.001)$, children's outside play $(\beta$ $=0.185, \mathrm{p}=.001)$, and children's lessons $(\beta=-0.052, \mathrm{p}=.032)$ (Positive values represent increases during the pandemic compared to before the pandemic). We found a significant interaction between period and age in prosocial behavior $(\beta=0.080, \mathrm{p}=.001)$. The effects of period were significant in 5 - $(\beta=1.000, p=.009)$ and 7-year-old $(\beta=0.843, p=.036)$ children. Children's prosocial behavior and peer problems are displayed as a function of age in Figure 1. 
163 Next, we conducted SEM analyses to assess whether the effects of period and children's age on peer

164 problems and prosocial behavior were mediated by differences in children's social lives. Specifically, we used durations of children's schooling, children's outside play, and children's lessons as mediation variables and children's sleeping time as a control variable (Figure 2). We selected the model that included direct paths between period and peer problems and between interaction and prosocial behavior $(\chi 2=62.304$, RMSEA $=.047, \mathrm{CFI}=.966)$ because fit indices indicated that it provided a better fit to the data than a model without the direct path $(\chi 2=80.494$, RMSEA $=.053$, $\mathrm{CFI}=.952)$. In this model, period was positively $(\beta=0.491, \mathrm{p}=.022)$ and negatively $(\beta=-2.843, \mathrm{p}$ $<.001)$ associated with durations of outside play and schooling, respectively. Interaction between age and period negatively associated with schooling $(\beta=-0.214, \mathrm{p}<.001)$. In addition, the duration of play negatively associated with peer problems $(\beta=-0.261, p=.001)$. However, schooling was not significantly associated with prosocial behavior $(\beta=0.113, \mathrm{p}=.070)$.

Finally, we evaluated the mediation effects of outside play on the relationship between

\section{Discussion}

180 The results revealed that peer problems and prosocial behavior, but not emotional symptoms, conduct problems, hyperactivity, differed between before and during the pandemic. Although there were no mediation effects on the relationship between period and prosocial behavior, we found an interaction effect between the pandemic and age in prosocial behavior. The results were partially consistent with our hypothesis that age may modulate the effect of the pandemic on prosocial behavior. Specifically, 4-year-old children scored equally before and during the pandemic, but older children showed more prosocial behavior during the pandemic compared to those before it. One possible interpretation for the increase in prosocial behavior was in-group favoritism. Items used to assess prosocial behavior included children's behavior towards in-group members, such as parents, siblings, or peers. Research on the behavioral immune system suggests that a pathogen infection can induce in-group favoritism and out-group aversion (Ackeerman et al., 2018). The behavioral immune system refers to a motivational system that helps minimize infection risk by changing cognition, affect, and behavior to avoid infection with a pathogen. It has been consistently reported that the behavioral immune system in individuals at risk of infection facilitates stereotypes and prejudicial attitudes toward outgroup members and increases in-group favoritism, such as greater conformity to social norms and increased collectivism (Murray \& Schaller, 2012; Wu \& Chang, 2012). Such in-group favoritism may motivate cognitions and behaviors for the avoidance of novel parasites contained in out-groups and for the management of local infectious disease (Thornhill \& Fincher, 2014; Wu \& Chang, 2012). Thus, children in this study may have increased prosocial behavior toward in-group members at the risk of pathogen infection to avoid infection by out-group members.

Children also showed more problems in their peer relationships during than before the pandemic. Although we found a mediation effect of outside play, the direct effect between the pandemic and peer problems was larger. Other factors, such as level of children's stress, can mediate the relationship between the pandemic and peer problems. Nevertheless, we need to be careful about the interpretation of the results, because some items in peer problem (e.g., "tends to play alone" ) could be increased during the pandemic compared to before pandemic unless children played with siblings as much as they used to play with peers, and the increased scores did not necessarily mean the 
207 children were having trouble with peers. Taken together, our results showed that children's some of

208 the socio-emotional behaviors differed before and during the pandemic.

209 Our results showed that children exhibited better and worse socio-emotional behaviors during the

210 COVID-19 pandemic compared to before the pandemic. To our knowledge this is one of the first

211 studies to conduct a pre- and post-assessment of the impact of the COVID-19 pandemic on children's

212 behaviors. Although we assessed children's behavior using an online questionnaire, most of the

213 available research did not utilize the same method of assessment both before and during the

214 pandemic, which results in the difficulty of not having a valid comparison group. It is possible that

215 parents' answers to the surveys can reflect differences in parents, not in children, and we need to be

216 careful about the interpretations of the results. Nevertheless, we believe that web-based surveys may

217 be one of the best methods for addressing the effects of the pandemic on child development.

218 Another limitation in this study was that we compared the different sample before and the during

219 pandemic. We matched several background information that may affect socio-emotional behaviors

220

221

222

223

224

225

226 across samples, but we need to conduct longitudinal research to examine how children change their behaviors across different time points. Moreover, it remains unclear whether the results from this population can be generalized to other populations, because the growth rate in the number of infected persons and deaths in Japan was lower than in other countries (Worldmeter, 2020). Moreover, children's social-emotional development could be more severely impaired due to the pandemic, particularly if this difficult situation continues for a long period. Future research should address these issues.

\section{Conflict of Interest}

228 The authors declare that the research was conducted in the absence of any commercial or financial

229 relationships that could be construed as a potential conflict of interest.

\section{$230 \quad 6 \quad$ Author Contributions}

231 YM and NY developed the study concept. All authors contributed to the study design. Data 232 collection was performed by YM. All authors performed the data analysis and interpretation. YM

233 drafted the manuscript. CS, XM, and NY revised the manuscript. All authors approved the final

234 version of the manuscript for submission.

\section{Funding}

236 This research was supported by grants from JSPS to the first author.

\section{$237 \quad 8 \quad$ Acknowledgments}

238 We thank Chika Harada and Nobuhiro Mihune for helpful comments on an earlier version of the

239 manuscript. This manuscript has been released as a pre-print at

\section{References}

241 Ackerman, J.M., Hill, S.E., and Murray, D.R. (2018). The behavioural immune system: Current

242 concerns and future directions. Soc Personal Psychol Compass. 12(2), e12371.

243 Bronfenbrenner, U. (1979). The Ecology of Human Development. Cambridge: Harvard Univ. Press. 
244 Dong, Y., Mo, X., Hu, Y., Qi, X., Jiang, F., Jiang, Z., et al. (2020) Epidemiology of COVID-19

245 among children in China. Pediatrics. 145, e20200702.

246 Golberstein, E., Wen, H., and Miller, B.F. (2020). Coronavirus disease 2019 (COVID-19) and mental health for children and adolescents. JAMA Pediatr. 174(9), 819-820.

248 Goodman, R. (1997). The Strengths and Difficulties Questionnaire: A research note. J Child Psychol 249 Psychiatr. 38(5), 581-586.

Matsuishi, T., Nagano, M., Araki, Y., Tanaka, Y., Iwasaki, M., Yamashita, Y., et al. (2008). Scale properties of the Japanese version of the Strengths and Difficulties Questionnaire (SDQ): a study of infant and school children in community samples. Brain Dev. 30(6), 410-415.

Ministry of Education, Culture, Sports, Science and Technology. (2020). Report of opening schools in the wake of the novel coronavirus pandemic in Japan. https://www.mext.go.jp/content/20200413mxt_kouhou01-000006421_1.pdf[Accessed September 13, 2020]. executive function during early childhood: A longitudinal study. European Journal of Dev Psychol. $17(3), 352-364$.

259 Murray, D.R., and Schaller, M. (2012). Threat(s) and conformity deconstructed: Perceived threat of 260 infectious disease and its implications for conformist attitudes and behavior. Eur J Soc Psychol. $26142(2), 180-188$.

Orgilés M. et al. (2020). Immediate psychological effects of the COVID-19 quarantine in youth from Italy and Spain. Preprint PsyArXiv [Preprint]. Available at: https://psyarxiv.com/5bpfz/ (Accessed

264 September 13, 2020).

265 Pathak, E.B., Salemi, J.L., Sobers, N., Menard, J., and Hambleton, I.R. (2020). COVID-19 in children in the United States: Intensive care admissions, estimated total infected, and projected numbers of severe pediatric cases in 2020. J Public Health Manag Pract. 26(4), 325-333.

268 Pisano, L., Galimi, D., and Cerniglia, L. (2020). A qualitative report on exploratory data on the 269 possible emotional/behavioral correlates of COVID-19 lockdown in 4-10 years children in Italy.

270 Preprint PsyArXiv [Preprint]. Available at: https://psyarxiv.com/stwbn/ (Accessed April 13, 2020).

271 Rosseel, Y. (2012). lavaan: An R package for structural equation modeling. J Stat Softw. 48(2), 1-36.

272 Thornhill, R., and Fincher, C.L. (2014). The parasite-stress theory of sociality, the behavioural 273 immune system, and human social and cognitive uniqueness. Evol Behav Sci. 8(4), 257-264.

274 World Health Organization. (2020). Coronavirus disease (COVID-19) advice for the public. 275 https://www.who.int/emergencies/diseases/novel-coronavirus-2019/advice-for-public. (Accessed 276 April 29, 2020).

277 Worldometer. COVID-19 coronovirus pandemic. https://www.worldometers.info/coronavirus/.

278 (Accessed May 14, 2020). 
Wu, B.P., and Chang, L. (2012). The social impact of pathogen threat: How disease salience influences conformity. Pers Individ Dif. 53(1), 50-54.

281 Wu, Z., and McGoogan, J.M. (2020). Characteristics of and important lessons from the coronavirus 282 disease 2019 (COVID-19) outbreak in China: Summary of a report of 72314 cases from the Chinese

283 Center for Disease Control and Prevention. JAMA. 323(13), 1239-1242.

284 Xie, X., Xue, Q., Zhou, Y., Zhu, K., Liu, Q., Zhang, J. et al. (2020). Mental health status among 285 children in home confinement during the coronavirus disease 2019 outbreak in Hubei Province,

286 China. JAMA Pediatr. doi: 10.1001/jamapediatrics.2020.1619

$287 \quad 10 \quad$ Data Availability Statement

288 The datasets [GENERATED/ANALYZED] for this study can be found in the [NAME OF

289 REPOSITORY] [LINK]. Please see the Data Availability section of the Author guidelines for more 290 details.

\section{$291 \quad 11$ Ethics Statement}

292 The study was conducted in accordance with the principles of the Declaration of Helsinki and the 293 procedure of the study was approved by the local ethics committee. Written informed consent 294 (including study purpose, methodology, risks, right to withdraw, duration of the experiment, handling 295 of personal information, and voluntary nature of participation) was obtained from all participating 296 parents prior to administering the survey. 


\begin{tabular}{|c|c|c|c|c|}
\hline \multirow[b]{2}{*}{ Parent Measure } & \multicolumn{2}{|c|}{ BEFORE $(\mathrm{N}=420)$} & \multicolumn{2}{|c|}{ DURING $(\mathrm{N}=420)$} \\
\hline & Mean & $\mathrm{SD}$ & Mean & SD \\
\hline Parent's age & 39.74 & 5.81 & 40.44 & 5.38 \\
\hline Number of family members & 3.99 & 0.94 & 4.04 & 1.05 \\
\hline Parental level of education & 3.22 & 0.89 & 3.17 & 0.91 \\
\hline \multicolumn{5}{|l|}{ Children Measure } \\
\hline Children's age in months & 83.37 & 20.37 & 83.95 & 20.90 \\
\hline Sleeping hours & 9.35 & 0.81 & 9.54 & 0.77 \\
\hline Days of schooling per week & 4.95 & 0.49 & 0.72 & 1.73 \\
\hline Hours of outside play per & 0.64 & 0.67 & 0.83 & 0.89 \\
\hline Hours of lessons per day & 0.26 & 0.35 & 0.21 & 0.37 \\
\hline Conduct problems & 2.45 & 1.73 & 2.37 & 1.82 \\
\hline Emotional symptoms & 2.10 & 2.11 & 2.19 & 2.14 \\
\hline Hyperactivity & 4.03 & 2.19 & 4.07 & 2.42 \\
\hline Peer problems & 2.24 & 1.75 & 2.50 & 1.84 \\
\hline Prosocial behavior & 5.38 & 2.45 & 5.90 & 2.43 \\
\hline Categorical Measure & $\%$ & & $\%$ & \\
\hline Children's sex (ratio of & $50 \%$ & & $50 \%$ & \\
\hline Respondent (ratio of & $91 \%$ & & $88 \%$ & \\
\hline
\end{tabular}

311

312

313

314

315

316

317

318

319 
320 Figure Legends.

321 Figure 1. Children's prosocial behavior and peer problems as a function of age

322 Figure 2. Effects of pandemic (period), age and the interaction on children's prosocial behavior and

323 peer problems. We included sleeping time, children's sex, parental age, the number of family

324 members, and parental education as control variables. For visibility, the non-significant paths, error

325 variances, and covariances were removed and reported in Table S2. * $\mathrm{p}<.05, * * \mathrm{p}<.01, * * * \mathrm{p}$

$326<.001$ 